\title{
A Germin-Like Protein Gene (CchGLP) of Capsicum chinense Jacq. Is Induced during Incompatible Interactions and Displays Mn-Superoxide Dismutase Activity
}

Fabiola León-Galván ${ }^{1}$, Ahuizolt de Jesús Joaquín-Ramos ${ }^{2,3}$, Irineo Torres-Pacheco ${ }^{4}$, Ana P. Barba de la Rosa ${ }^{2}$, Lorenzo Guevara-Olvera ${ }^{3}$, Mario M. González-Chavira ${ }^{\mathbf{5}}$, Rosalía V. Ocampo-Velazquez ${ }^{4}$, Enrique Rico-García ${ }^{4}$ and Ramón Gerardo Guevara-González ${ }^{4, *}$

1 División de Ciencias de la Vida, Departamento de Ingeniería en Alimentos, Campus Irapuato-Salamanca, Universidad de Guanajuato, Ex-Hacienda el Copal km 9, Carretera Irapuato-Silao, CP 36500, Irapuato Guanajuato, Mexico; E-Mail: ingfaby@yahoo.com.mx

2 División de Biología Molecular, Instituto Potosino de Investigación Científica y Tecnológica, Camino a la Presa San José 2055, Colima, Lomas 4 sección CP 78216, San Luis Potosí, S.L.P. Mexico; E-Mails: ahuizolt_j@hotmail.com (A.J.J.-R.); apbarba@ipicyt.edu.mx (A.P.B.R.)

3 Laboratorio de Biología Molecular, Departamento de Ingeniería Bioquímica, Instituto Tecnológico de Celaya, Avenida Tecnológico y A, García-Cubas, S/N, Col, FOVISSSTE, CP 38010, Celaya, Guanajuato, Mexico; E-Mail: lorenzogo@yahoo.com

4 C.A de Ingeniería de Biosistemas, Facultad de Ingeniería, Universidad Autónoma de Querétaro, Centro Universitario Cerro de las Campanas, S/N, Col, Las Campanas, CP 76010, Santiago de Querétaro, Querétaro, Mexico; E-Mails: torresirineo@gmail.com (I.T.-P.); rosov05@yahoo.com.mx (R.V.O.-V.); ricog@uaq.mx (E.R.-G.)

5 Unidad de Biotecnología, Instituto Nacional de Investigaciones Forestales, Agrícolas y Pecuarias, Carr, Celaya-San Miguel de Allende, km 6, CP 38010, Celaya, Guanajuato, Mexico; E-Mail: gonzalez.mario@inifap.gob.mx

* Author to whom correspondence should be addressed; E-Mail: ramon.guevara@uaq.mx; Tel.: +52-442-192-1200; Fax: +52-442-192-1200 (ext.6006).

Received: 21 July 2011; in revised form: 4 September 2011 / Accepted: 17 October 2011 / Published: 25 October 2011

Abstract: A germin-like gene (CchGLP) cloned from geminivirus-resistant pepper (Capsicum chinense Jacq. Line BG-3821) was characterized and the enzymatic activity of the expressed protein analyzed. The predicted protein consists of 203 amino acids, similar to other germin-like proteins. A highly conserved cupin domain and typical germin boxes, 
one of them containing three histidines and one glutamate, are also present in CchGLP. A signal peptide was predicted in the first $18 \mathrm{~N}$-terminal amino acids, as well as one putative $N$-glycosylation site from residues 44-47. CchGLP was expressed in E. coli and the recombinant protein displayed manganese superoxide dismutase (Mn-SOD) activity. Molecular analysis showed that CchGLP is present in one copy in the C. chinense Jacq. genome and was induced in plants by ethylene (Et) and salicylic acid (SA) but not jasmonic acid (JA) applications in the absence of pathogens. Meanwhile, incompatible interactions with either Pepper golden mosaic virus (PepGMV) or Pepper huasteco yellow vein virus (PHYVV) caused local and systemic CchGLP induction in these geminivirus-resistant plants, but not in a susceptible accession. Compatible interactions with PHYVV, PepGMV and oomycete Phytophthora capsici did not induce CchGLP expression. Thus, these results indicate that $C \operatorname{ch} G L P$ encodes a Mn-SOD, which is induced in the $C$. chinense geminivirus-resistant line BG-3821, likely using SA and Et signaling pathways during incompatible interactions with geminiviruses PepGMV and PHYVV.

Keywords: GLP; geminiviruses; Mn-SOD; ethylene; salicylic acid

\section{Introduction}

Germins and germin-like proteins (GLPs) have been implicated in the reinforcement of plant cell wall strength providing plants with resistance to biotic and abiotic stresses [1-3]. A key feature of the GLP-related subfamilies, including germins, is the presence of germin boxes and conservation of a cupin superfamily (beta-barrel proteins) derived-motif [4-7]. This motif contains three highly conserved histidines and one glutamate residue involved in binding a metal ion [8]. GLPs have been classified into subfamilies based on having oxalate oxidase (OXO) or superoxide dismutase (SOD) activities [7-10]. OXO and SOD activities result in production of hydrogen peroxide. A new GLP gene (hereafter named as $C c h G L P$ ) was previously identified in a geminivirus-resistant Capsicum chinense line named BG-3821 [11,12]. CchGLP is specifically induced in incompatible interactions of $C$. chinense BG-3821 with pepper huasteco yellow vein virus (PHYVV), Xanthomonas campestris pv. vesicatoria and Fusarium solani [11]. According to classification suggested by Park et al. [2] and based on this expression pattern in different plant-microbe interactions, CchGLP was considered a pathogenesis-related (PR) protein of the family PR-16 [11]. PR proteins are defined as host plant proteins induced specifically in pathological or related situations. They are not accumulated locally in the infected leaf, but are also systemically induced, associated with the development of systemic acquired resistance (SAR) against further infection by fungi, bacteria and viruses [13]. In order to characterize in more detail the possible role of CchGLP in pathogen resistance in C. chinense BG-3821, it is necessary to evaluate gene expression in other plant-pathogen interactions as well as molecular aspects of this gene and the functional nature of the protein. Therefore, the aim of this work was to characterize the CchGLP gene at the molecular level and the enzymatic activity of the expressed recombinant protein, as well as to evaluate $C c h G L P$ expression in different compatible and incompatible interactions. The deduced amino acid sequence of the CchGLP gene contains a signal peptide, conserved histidines and 
glutamate residues as well as a germin boxes, as reported for other GLPs. Moreover, a manganese superoxide dismutase (Mn-SOD) activity for the CchGLP protein expressed in E. coli was detected. Molecular studies revealed that $C$ chGLP is present in one copy in the genome. In addition, CchGLP was locally and systemically induced during incompatible interactions with pepper golden mosaic virus (PepGMV) and after ethylene and salicylic acid applications in the absence of pathogen in this geminivirus-resistant line. Several implications of our results regarding the plant-microbe interactions are discussed.

\section{Results and Discussion}

\subsection{Characterization of the CchGLP Sequence}

The $C$ chGLP cDNA sequence contains a 24 bp leader section, poly A tail, and an ORF encoding a predicted protein of 203 amino acids. Figure 1 shows a phylogenetic tree of amino acid sequences from several solanaceae and Arabidopsis thaliana plant proteins, which according to BLAST analysis were the most related to CchGLP. The deduced protein of CchGLP was more related to a GLP of Nicotiana tabacum (93\% identity, accession number: BAH15357).

Figure 1. Phylogenetic tree of CchGLP amino acid sequences and other plant GLPs. Accession numbers of GLP amino acid sequences evaluated in the tree are: $C$ chinense (ABG76199), A. thaliana (NP_177405), Auxin binding protein from Solanum nigrum (ADW66138), GLP $24 \mathrm{~K}$ from Nicotiana tabacum (BAC77634), GLP from Nicotiana tabacum (BAH15357), Auxin binding protein from Solanum nigrum (ADW66150) and auxin binding protein 19a from Prunus persica (Q9ZRA4).

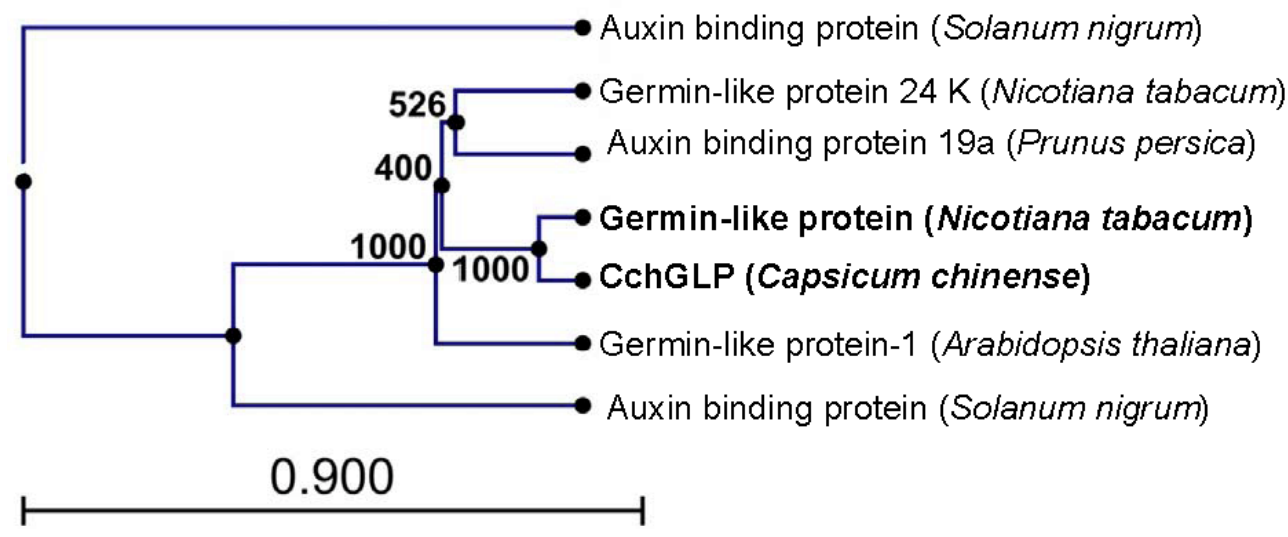

The CchGLP protein sequence was submitted to bioinformatic analysis in order to characterize it in more detail. A highly conserved cupin 2 domain (not shown) and structure that contains three boxes representing the germin domains according to $\mathrm{Lu}$ et al. [14] are present in CchGLP (Figure 2). In addition, $C$ ch $G L P$ was predicted to contain a $N$-terminal signal peptide of 18 amino acids, and one potential $N$-glycosylation site from amino acids 44-47 (NVTV) (Figure 2). Moreover, a putatively important sequence KGD (or sometimes KGE) that has been detected in over $50 \%$ of GLPs, but not in germins was detected in CchGLP (Figure 2). 
The number of amino acids in the deduced protein as well as sequence features were typical of those reported in plant GLPs elsewhere [2,4,6]. Glycosilation in germins appears to be essential in protein-protein interactions but not for enzyme activity as demonstrated by Pan et al. [15].

Figure 2. Amino acid sequence alignment of CchGLP and GLPs from some Solanaceae and $A$. thaliana. Amino acids forming the germin boxes are indicated with solid squares. The long dashed rectangle in the $N$-terminus indicates amino acids of predicted signal peptide. The short dashed rectangle shows a predicted $N$-glycosylation site (NVTV). The red line shows the tripeptide KGD (or sometimes KGE), characteristic of almost $50 \%$ of GLPs. Asterisk display important invariable amino acids that are conserved in GLPs. Yellow highlighting displays conserved amino acid residues among aligned GLPs.

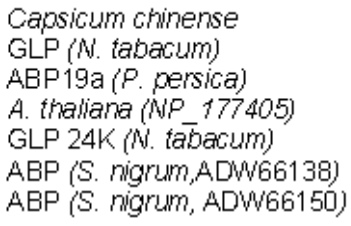

Capsicum chinense GLP (N. tabacum) ABP19a ( $P$. persica) A. thaliana (NP 177405) GLP 24K (N. tabacum) ABP (S. nigrum,ADW66138) ABP (S. nigrum, ADW66150)

Capsicum chinense GLP (N. tabacum) ABP19a ( $P$. persica) A. thaliana (NP_ 177405) GLP $24 \mathrm{~K}$ (N tabacum) ABP (S. nigrum,A.DW66138) ABP (S. nigrum, ADW66150)

Capsicum chinense GLP ( $N$ tabacum) ABP19a ( $P$. persica) A. thaliana (NP 177405) GLP 24K (N. tabacum) ABP (S. nigrum,ADW66138) ABP (S. nigrum, ADW66150)

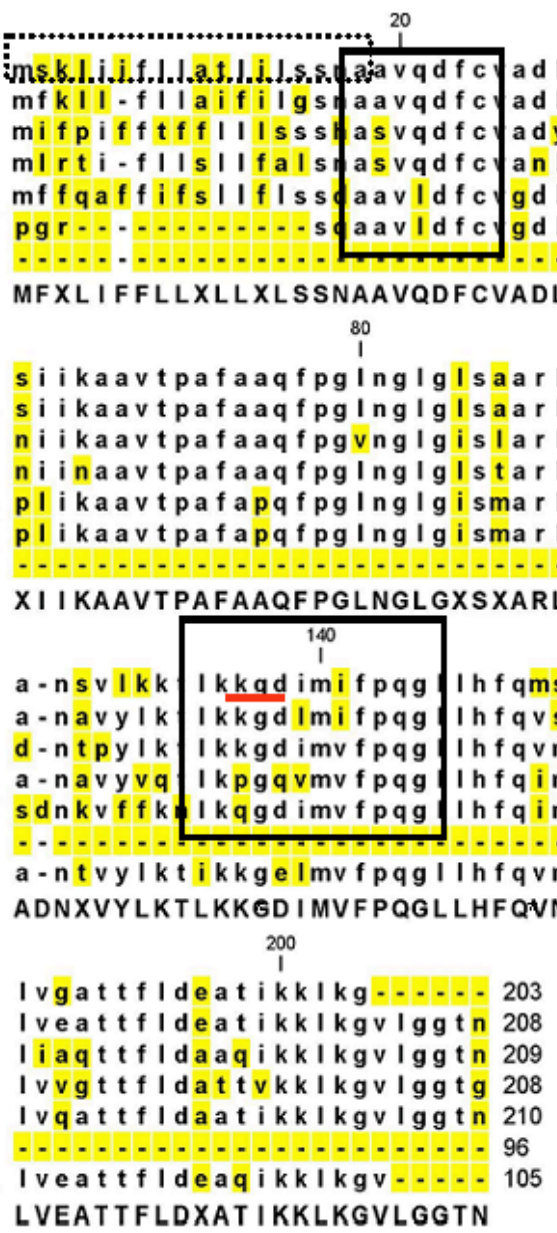

40 ad I kapespcgyscksvtty y ydf $v$ fsg Is a a n t a 62 ad I kgpespagys cksvanvt vdd f v f sg Is a agnt a 61 ady k a pdgagys ck kpakvt ind f vysg I g i agnt t 62 an I k raetpagypcirpihvkatdf vfsg Igtpgntt 61 gd Isvpdgpggyackkpsavtand fvfsglatpvkIn 62 gd I s I pdgpcgysckkpskvtaddf vf sg I a t pvk In 50

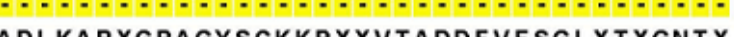

120

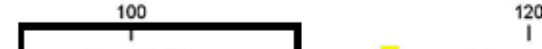

plg vipfhthpgasev I I vtqgsitagfvss 124 v I I vvqgsitagf vs 123 e v I I vvqgt i iagf vas 124 v IfvItgsitagf vs 123 v I yvvtge icagfis s 124

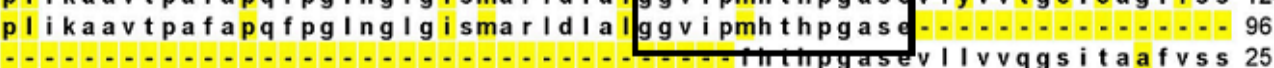

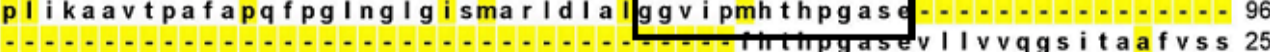
XI I KAAVTPAFAAQFPGLNGLGXSXARLDLAPGGV I PFHTHPGASEVLLVVQGS I TAĢ FVS 160 180

g f vf f sssug I q i t d f a I f and I pt k 185

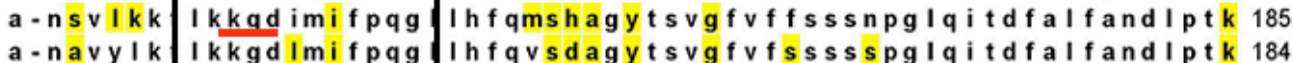
d-ntpy I k I k kgdimvfpqg I h fqungggtpalafpsfsspspg Iqi Idfalfknd I pte 185 a-navyvq I kpgqvmvfpqg Ihfqinagkssasavvtfnsanpg Iqi Idfalfans I pae 184 sdnkvffk I kqgdimvfpqg I hfqinsgkttglaivsfssptpglqitdfalfand Iate 186

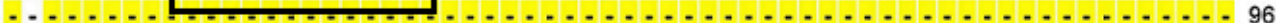
a-ntvy I kt ikkge Imvfpqg I I hfqvnavgfnsvayvffsssnpglqitdfal fand Ist k 86

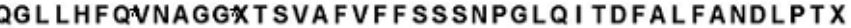

I vgat t f I deat ikkikg.......203

Iveat t f I deat ikk I kgv Iggt n 208 I i aqt t f I daaq i k k I kgv I g g t n 209

( ................................ 96

LVEAT TFLDXAT I KKLKGVLGGTN

\subsection{CchGLP Protein Purification}

To carry out a more detailed analysis of CchGLP, the recombinant protein was produced in the E. coli system. A protein with the expected molecular weight for CchGLP protein without the signal peptide $(20.5 \mathrm{kDa})$ was mainly detected in the soluble fraction (over $20 \%$ of total proteins). The recombinant protein was observed $4 \mathrm{~h}$ after IPTG induction, but was not observed in the control test without induction (Figure 3A). Recombinant CchGLP protein was further verified with Western blot analysis using specific antibodies against the His-tail tag added to the protein (Figure 3B). After purification of the recombinant protein through Ni-columns, the elution fractions showed a band of 
$20.5 \mathrm{kDa}$ with a purity of above 95\% (Figure 3C). This purified protein was used for enzymatic activity determinations. It is worth mentioning that in contrast to reported problems of expression of germin proteins in E. coli due to protein aggregation [16], in this work we did not have these problems that appeared to reduce expression in this system.

Figure 3. In vitro CchGLP protein production in E. coli. Panel A, SDS-PAGE displaying CchGLP (Germin-Like protein) produced during $24 \mathrm{~h}$ post-IPTG induction. Lane 1, negative control [proteins extracted from E. coli transformed with plasmid pET22b $(+)$ ]; Lane 2, molecular size marker; Lanes 3-10, proteins obtained in E. coli transformed with plasmid pET22b (+)-CchGLP at 4, 7, 10, 13, 16, 19, 24 and $0 \mathrm{~h}$ post-IPTG induction. Panel $\mathrm{B}$, Western blot analysis for recombinant protein production in E. coli transformed with plasmids harboring or not the full-length CchGLP cDNA sequence. Lane 1, negative control; Lanes 2-9, protein extracts from E. coli at 24, 19, 16, 13, 10, 7, 4 and $0 \mathrm{~h}$ post-IPTG induction. Antibody used was specific against the His tail engineered in the recombinant protein (see Materials and Methods). Panel C, Lane 1 molecular size marker; Lane 2, CchGLP (germin-like protein) recombinant protein purified using Ni-column. In all panels, arrows indicate the presence of CchGLP. Molecular size marker used was Benchmark $^{\mathrm{TM}}$ (Invitrogen).

A

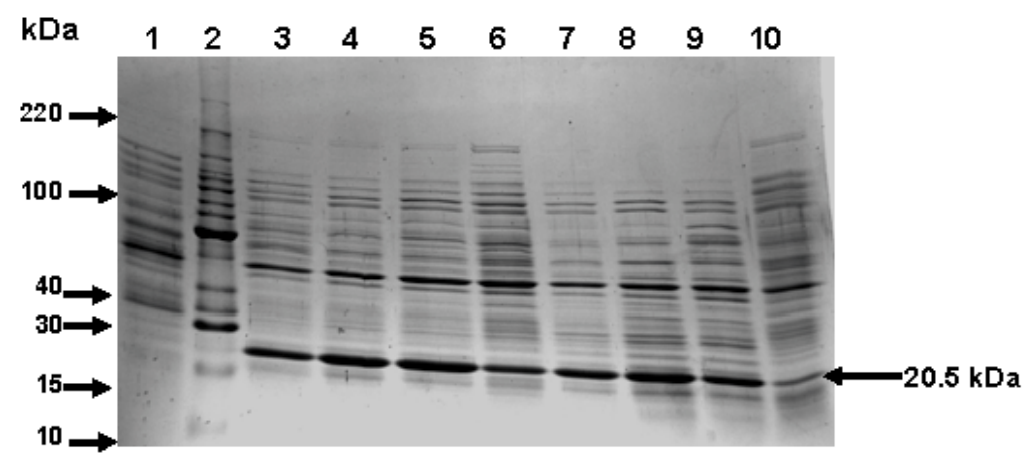

B

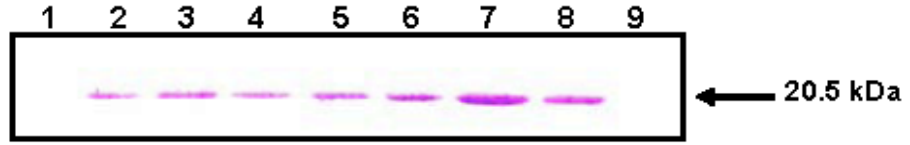

C

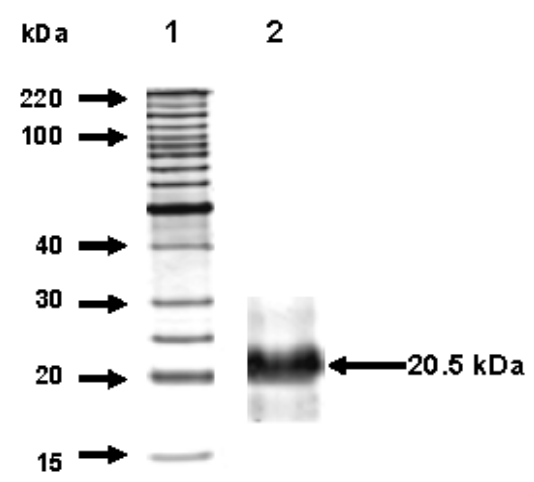




\subsection{In Vitro Enzymatic Activity of CchGLP}

Proteins belonging to the group of "germins" show an OXO activity mainly related to their role in defense [2]. On the other hand, "germin-like" proteins can also accumulate during pathogen attack although they are totally devoid of OXO but display SOD activity [2]. Moreover, several germin proteins expressed in Pichia pastoris might form multimers which are required for activity [15] and homohexameric barley germin, for which the 3D structure has been solved, displays both OXO and SOD activities [17,18]. Thus, in order to determine whether CchGLP displayed either OXO or SOD activity (or both activities), these assays were carried out. OXO activity was not detected for CchGLP (data not shown), instead the CchGLP purified protein displayed SOD activity (Figure 4A). Inhibition assays of SOD activity using either $\mathrm{H}_{2} \mathrm{O}_{2}$ (inhibits Fe-SOD activity) or $\mathrm{KCN}$ (inhibits $\mathrm{Cu} / \mathrm{Zn}-\mathrm{SOD}$ activity) are used to identify the type of SOD activity [19]. On the other hand, Mn-SOD activities are resistant to both compounds [19]. Thus, inhibition assays of SOD activity revealed that CchGLP was not inhibited by either compound, indicating Mn-SOD activity for this protein (Figure 4B,C).

Figure 4. Enzymatic activity of $C c h G L P$ recombinant protein. Panel A, SOD activity determination. Lane 1, negative control ( $25 \mu \mathrm{g}$ of purified tannase from A. niger); Lanes 2 and 3, 25 and $15 \mu \mathrm{g}$ of purified CchGLP protein, respectively; Lane 4, bovine erythrocyte superoxide dismutase $(15 \mu \mathrm{g})$. Panel B, Effect of $\mathrm{H}_{2} \mathrm{O}_{2}$ on $C c h G L P$ activity. Lanes $1-4,25$, 20, 15 and $10 \mu \mathrm{g}$ of purified CchGLP protein; Lane 5, positive control (10 IU of commercial Fe-SOD from E. coli). Panel C, Effect of KCN on CchGLP activity. Lanes 1-4, 10, 15, 20 and $25 \mu \mathrm{g}$ of purified CchGLP protein; Lane 5, positive control (10 IU of bovine erythrocyte $\mathrm{Cu} / \mathrm{Zn}-\mathrm{SOD})$.

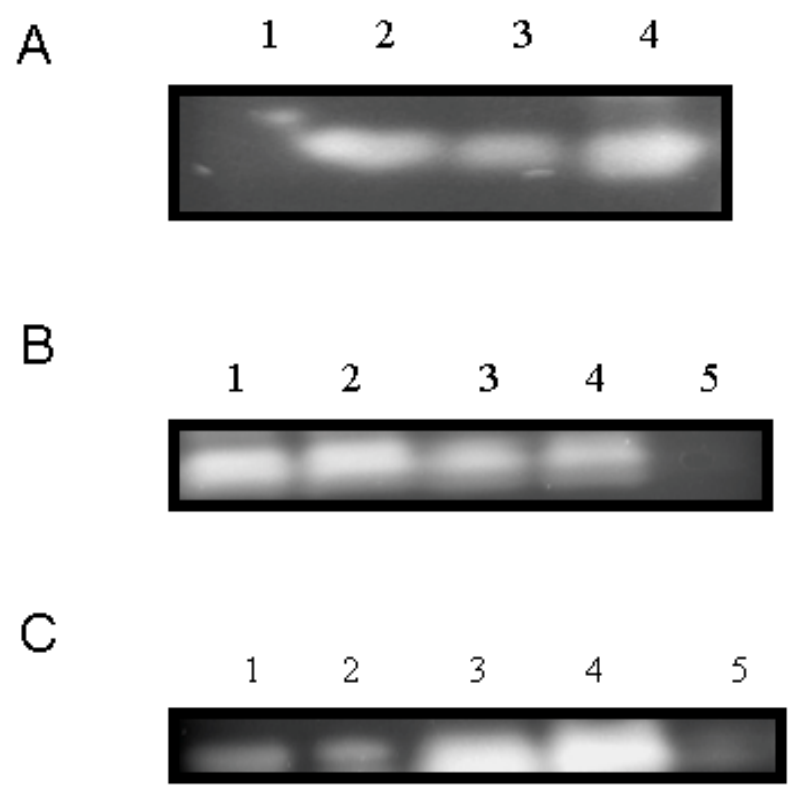

Plant Mn-SODs have been reported to occur extracellularly as well as in mitochondria and peroxisomes $[5,18]$. Moreover, Mn-SOD activities have previously been associated with defense against biotic stress in plants $[13,18]$. 


\subsection{Molecular Characterization and Gene Expression of CchGLP during Plant-Microbe Interactions}

The CchGLP cDNA sequence was compared with a genomic sequence obtained using PCR with specific oligonucleotides. No differences were detected between the sequences, indicating no introns are present in the CchGLP genomic sequence. Hybridization assays using total genomic DNA from C. chinense BG-3821 (geminivirus-resistant) and UX-SMH-55 (geminivirus-susceptible, data not shown), showed either a single or two bands when genomic DNA was digested with restriction enzymes cutting outside or once inside the CchGLP gene, respectively (Figure 5A). CchGLP was not detected in hybridization assays with Capsicum annuum cv. "Sonora Anaheim" genomic DNA (data not shown). These results indicated a single copy of the CchGLP gene in both $C$. chinense accessions, and the absence of the CChGLP gene in C. annuum. On the other hand, CchGLP expression was studied in $C$. chinense BG-3821 during compatible and incompatible interaction with Pepper golden mosaic geminivirus (PepGMV) as well as two plant-pathogen signal molecules, namely ethylene and jasmonic acid. It was observed that CchGLP was induced at least from $12 \mathrm{~h}$ to $48 \mathrm{~h}$ in inoculated leaves post-inoculation or application, either by PepGMV, PHYVV, salicylic acid or ethylene (Figure 5B,C). CchGLP induction was also observed in newly emerged systemically geminivirus-infected leaves 15 days post-inoculation (Figure 5B). On the other hand, neither $P$. capsici infection nor jasmonic acid application induced CchGLP expression in C. chinense BG-3821 (Figure 5B,C). Compatible interaction in a geminivirus-susceptible $C$. chinense accession (UX-SMH-55) did not induce $C c h G L P$ when inoculated neither with PepGMV, PHYVV, P. capsici nor Et, JA or SA applications (not shown).

Several lines of evidence shows that GLPs are important in plant protection against pathogens and pests, or induced with applications of chemicals such as salicylic acid, ethylene or hydrogen peroxide [20-22]. A previous report showed that CchGLP was induced in incompatible interactions between $C$. chinense BG-3821 and PHYVV, Xanthomonas campestris pv. vesicatoria and not in a compatible interaction with oomycete Phytophthora capsici [11]. In this study, it was shown that $C c h G L P$ was also induced in incompatible, but not in compatible, interactions with another geminivirus affecting pepper in Mexico (PepGMV) in C. chinense accessions. The phenotype of susceptibility to geminiviruses in $C$. chinense UX-SMH-55 evaluated, might, at least partially, be explained by the absence of CchGLP induction in all infections and plant signal molecules applications evaluated. Defense response genes are also uninduced in other susceptible plants as showed elsewhere $[2,11,12]$. The fact that the CchGLP gene was not detected in the genome of the geminivirus-susceptible $C$. annuum cv. "S. Anaheim", suggest either a low homology with this gene or absence in the genome of this pepper species, as occurred with other genes previously identified in C. chinense BG-3821 [12]. Moreover, CchGLP was also demonstrated to be induced by SA and Et applications in the absence of pathogens [11]. In this study, it was demonstrated that Et but not JA induced local and systemic CchGLP gene expression in the absence of pathogens. The SA and JA signaling pathways are mutually antagonistic, whereas SA and Et pathways appear to interact $[23,24]$. Further studies are necessary to determine the role of this signal routes in this plant-microbe interaction. 
Figure 5. Determination of genomic copy number and expression profile with several inducers of CchGLP. Panel A, C. chinense BG-3821 genomic DNA was digested with either Pst I (cut inside CchGLP gene), Hind III (cut outside CchGLP gene) or Eco RI (cut outside CchGLP gene) enzymes. Lane 1, positive control (5 $\mu \mathrm{g}$ of linearized plasmid PCR4.0-CchGLP Eco RI digested); Lanes 2-4 signal displayed of $20 \mu \mathrm{g}$ of $C$. chinense BG-3821 genomic DNA digested with either, Pst I, Hind III and Eco RI, respectively. Panel B, CchGLP gene induction in C. chinense BG-3821 inoculated leaves after 12, 24, 36 and 48 h post-infection; NE, newly emerged leaf; Mock, mock-inoculated leaf with plasmid bluescript SK+ (geminivirus) or uninoculated potato dextrose agar blocks (P. capsici). Panel C, the same order of lanes as in panel B, but using plant growth regulators in the absence of geminiviruses or oomycetes as possible CchGLP inducers. Mock-inoculation was carried out with deionized water. In all slots, $15 \mu \mathrm{g}$ of total RNA were loaded. The complete ORF sequence of CchGLP was used as a probe in all cases. $\mathrm{C}-$ and $\mathrm{C}+$, are negative and positive controls of hybridization, using $C$. annuum genomic DNA and plasmid PCR4.0-CchGLP, respectively.

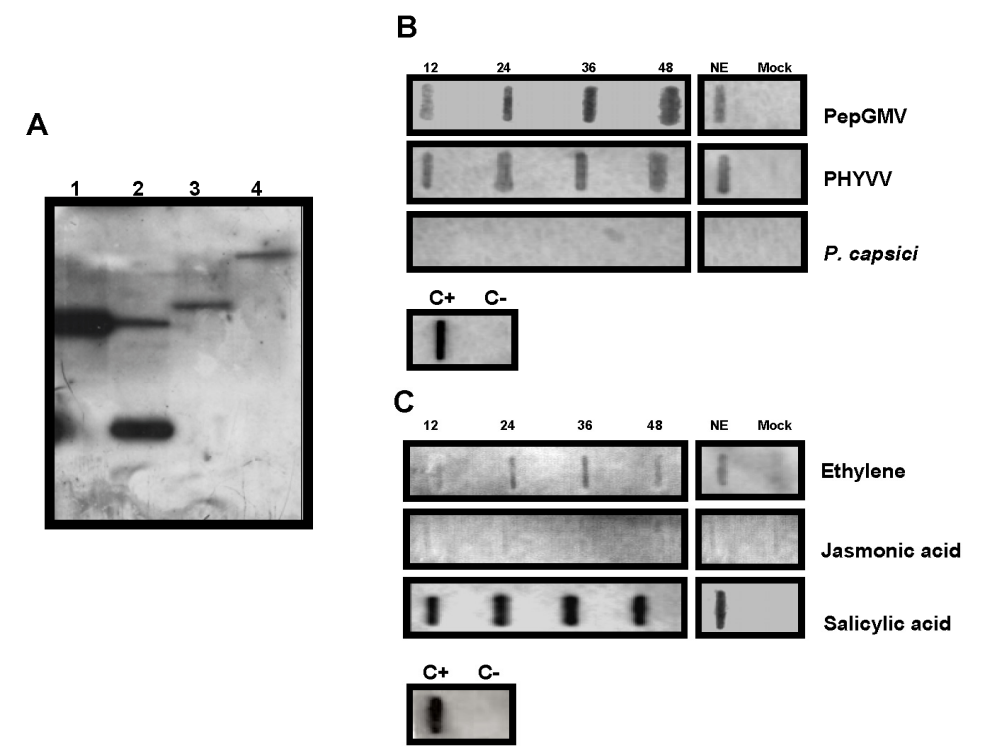

\section{Experimental Section}

\subsection{CchGLP Cloning for Protein Expression in E. coli}

Based upon the CchGLP sequence (accession number DQ677335), two oligonucleotides to amplify the $C c h G L P$ open reading frame without signal peptide were designed. Oligonucleotide sequences included restriction sites for $M s c$ I and Xho I enzymes in the forward and reverse primers, respectively. The sequence of these primers (restriction sites are written in lower case) was as follows: (Forward) 5'tggccaGCTGTTCAAGATTTCTGCGTCGC-3', (Reverse) 5'-gagctcCTTAATCGTAGCTTCATCAAGG-3'. PCR reaction mix contained the following components: $0.75 \mu \mathrm{L}$ each dNTPs $(2.5 \mathrm{mM}), 2 \mu \mathrm{L}$ of oligonucleotides $(50 \mathrm{ng} / \mathrm{mL}), 0.5 \mu \mathrm{L}$ of Taq DNA polymerase $(6 \mathrm{U} / \mu \mathrm{L}), 1 \mu \mathrm{L}$ of DNA from CchGLP cloned in $\mathrm{PCR}^{\mathrm{TM}} 4-\mathrm{TOPO}^{\mathrm{R}}$ (Invitrogen) in a reaction volume of $50 \mu \mathrm{L}$. PCR conditions to amplify the CchGLP gene were: $94^{\circ} \mathrm{C}, 1 \mathrm{~min} ; 51^{\circ} \mathrm{C}, 1 \mathrm{~min}$ and $72{ }^{\circ} \mathrm{C}, 2 \mathrm{~min}$ for 35 cycles. PCR products were 
visualized on $1.5 \%$ agarose gels using a digital image system (1D Image Analysis Software, version 3.02; Kodak Digital System, Rochester, NY, USA). This amplified product (containing Msc I and Xho I restriction sites) was purified from agarose gel using a gene clean commercial kit (QIAGEN, Mexico, $\mathrm{DF}, \mathrm{Mexico}$ ) and cloned into $\mathrm{PCR}^{\mathrm{TM}} 4-\mathrm{TOPO}^{\mathrm{R}}$. This construct was digested with the aforementioned enzymes and the CchGLP fragment was purified again from agarose gel. The pET22b (+) protein expression vector (Novagen), was digested with $M s c$ I and Xho I and then ligated with the CchGLP fragment using T4 ligase (Promega) for $3 \mathrm{~h}$ at $16{ }^{\circ} \mathrm{C}$. The resulting construct was named as $\mathrm{pET} 22 \mathrm{~b}(+)-C c h G L P$, and further evaluated using protein expression and enzymatic activity assays.

\subsection{CchGLP Production in E. coli}

A BL21 E. coli strain was transformed with pET22b (+)-CchGLP. Transformed cells were grown overnight at $32{ }^{\circ} \mathrm{C}$ in liquid Basal Salts-Glucose (BSG) medium [25]. Then, a $1 \mathrm{~mL}$ aliquot was inoculated into a flask with $100 \mathrm{~mL}$ of BSG medium with ampicillin $(50 \mathrm{mg} / \mathrm{mL})$ and $1 \mathrm{mM}$ of IPTG, and grown until an OD 0.6 of $32{ }^{\circ} \mathrm{C}$ was reached. Aliquots of $5 \mathrm{~mL}$ of this medium were evaluated from 3 to $24 \mathrm{~h}$ for GLP protein expression. Samples were centrifuged at 10,000 rpm for $10 \mathrm{~min}$ and then resuspended in $500 \mu \mathrm{L}$ saline phosphate buffer (SPB) [26]. Cells were disrupted using a sonicator (Ultrasonic processor) with an amplitude of $30 \%$ and $9.9 \mathrm{~s}$ pulses during $2 \mathrm{~min}$, then centrifuged at $10,000 \mathrm{rpm}$ for $10 \mathrm{~min}$. Soluble fraction was separated and resuspended in SPB. Protein quantification was carried out using the protein assay kit (Bio-Rad) [27]. Proteins were separated on SDS-PAGE gels (gradient from 4-20\%) and stained with Coomasie blue. The molecular size marker used was Benchmark $^{\mathrm{TM}}$ (Invitrogen). CchGLP protein purification under native conditions was carried out using affinity chromatography (ProBond, Invitrogen) following the manufacturer's recommendations and further evaluated by Western blot analysis using Anti-His ( $c$-term)-AP antibodies (Invitrogen).

\subsection{Determination of Enzymatic Activity}

Assays for OXO activity were carried out essentially as described in Dumas et al. [28]; the positive control was a commercial oxalate oxidase of barley (SIGMA-Aldrich). SOD activity was determined using the procedure reported by Beauchamp and Fridovich [29]. The gel was incubated in phosphate buffer $\mathrm{pH} 7.5$ containing $2.5 \mathrm{mM}$ of NBT, then the gel was rinsed in distilled water. Afterward, the gel was incubated in phosphate buffer containing $28 \mu \mathrm{M}$ of Riboflavine and $28 \mathrm{mM}$ TEMED for $20 \mathrm{~min}$, then rinsed with distilled water and placed on a soft light source. Determination of SOD type was carried out including inhibitory concentrations of either $\mathrm{H}_{2} \mathrm{O}_{2}$ or $\mathrm{KCN}$ when evaluating Fe-SOD or $\mathrm{Cu}-\mathrm{Zn}-\mathrm{SOD}$, respectively [19]. Commercial Fe-SOD from E. coli strain and bovine erythrocyte $\mathrm{Cu}-\mathrm{Zn}-\mathrm{SOD}$ were used as experimental controls (Grupo Nutramex, Mexico, DF, Mexico).

\subsection{Virus and Oomycete Inoculation and Plant Growth Regulators Applications}

Geminivirus-resistant (C. chinense line BG-3821) and susceptible (C. chinense line UX-SMH-55) plants were inoculated with $2 \mu \mathrm{g}$ of cloned viral DNA components (cloned in SK+ bluescript plasmid, Stratagene, La Jolla, CA, USA) using a biolistic procedure with a particle delivery system (Model PDS 1000, Dupont, Wilmington, DE, USA) as reported [30]. Both pepper lines were previously identified and 
characterized in our group based on resistance or susceptibility to geminivirus infections [30]. Before bombardments, PHYVV components A and B, and component B of PepGMV were excised from bluescript plasmid using HindIII. Component A of PepGMV was excised from the plasmid with the enzyme EcoRI in order to increase the percentage of plants infected using the biolistic procedure $[11,12,30]$. Plants were inoculated at the 4 leaf-stage and maintained in a greenhouse at $26-28{ }^{\circ} \mathrm{C}$ as reported [30]. Inoculation of $P$. capsici was performed according to Barrera-Pacheco et al. [11]. In all cases, newly emerged uninoculated leaves were sampled at 15 days post-inoculation to analyze systemic CchGLP gene induction. As controls, plants were mock-inoculated with the plasmid bluescript or potato dextrose agar blocks. PHYVV and P. capsici inoculations were used as positive or negative controls, respectively, for $C$ chGLP induction in $C$. chinense BG-3821 plants based on previous reports $[11,12,30]$. Applications of ethylene, jasmonic acid and salicylic acid were according to Barrera-Pacheco et al. [11]. Mock-inoculated plants for these treatments used water applications only.

\subsection{Hybridization Analysis}

The copy number of CchGLP in C. chinense BG-3821 was determined using standard Southern blotting protocols [26]. Gene induction studies of CchGLP in C. chinense BG-3821 and UX-SMH-55 were carried out by Northern blot (slot blot) as described in Barrera-Pacheco et al. [11]. A DNA fragment consisting of the complete ORF of CchGLP was used as a probe in both assays.

\subsection{Analysis of Genomic Sequence of CchGLP Structural Region}

Total genomic DNA was extracted following the procedure of Anaya-López et al. [12]. This DNA was used as a template for CchGLP structural region amplification using the specific oligonucleotides aforementioned in Section 3.1. The PCR amplified fragment was directly sequenced and compared to that from CchGLP cDNA, using bioinformatic tools (see below).

\subsection{Bioinformatic Analysis}

Analysis of GLP amino acid sequences was carried out using Lasergene 8.0 software (DNAstar). A phylogenetic tree was generated using the MegAlign tool of Lasergene 8.0. Accession numbers of GLP amino acid sequences evaluated in the tree are: C chinense (ABG76199), A. thaliana (NP_177405), Auxin binding protein from Solanum nigrum (ADW66138), GLP 24 K from Nicotiana tabacum (BAC77634), GLP from Nicotiana tabacum (BAH15357), Auxin binding protein from Solanum nigrum (ADW66150) and auxin binding protein 19a from Prunus persica (Q9ZRA4). Prediction of signal peptide and glycosylation sites was performed using ExPASy proteomics server tools [31].

\section{Conclusions}

The CchGLP gene encodes a germin-like protein with Mn-superoxide dismutase activity. This gene is induced during incompatible interactions and applications of plant defense inducer molecules such as SA and Et. Plant defense in response to microbial attack is regulated through a complex network of signaling pathways that involve three signaling molecules: SA, JA and Et. Taken together, our results 
support a possible role for CchGLP in pathogen resistance, and likely with SA and Et signal pathways involved in these events.

\section{Acknowledgments}

The authors thank SEP-CONACyT, PROMEP and FOMIX-Qro for supporting this research. I.T.P and BIBITEC also acknowledges to CONACyT-PROINOVA, for support in paying this paper. Special thanks to Angela Chapa-Oliver and Laura Mejia-Teniente for critical reading of the manuscript.

\section{References}

1. Schweizer, P.; Christoffel, A.; Dudler, R. Transient expression of members of the germin-like gene family in epidermal cells of wheat confers disease resistance. Plant J. 1999, 20, 541-552.

2. Park, C.-J.; An, J.-M.; Shin, Y.-Ch.; Kim, K.-J.; Lee, B.-J.; Paek, K.-H. Molecular characterization of pepper germin-like protein as the novel PR-16 family of pathogenesis-related proteins isolated during the resistance response to viral and bacterial infection. Planta 2004, 219, 797-806.

3. Gucciardo, S.; Wisniewski, J.-P.; Brewin, N.; Bornemann, S. A germin-like protein with superoxide dismutase activity in pea nodules with high protein sequence identity to a putative rhicadhesin receptor. J. Exp. Bot. 2007, 58, 1161-1171.

4. Dunwell, J.M.; Khuri, S.; Gane, P. Microbial relatives of the seed storage proteins of higher plants: Conservation of structure and diversification of function during evolution of the cupin superfamily. Microbiol. Mol. Biol. Rev. 2000, 64, 153-179.

5. Carter, C.; Thornburg, R. Tobacco nectarin I-purification and characterization as a germin-like, manganese superoxide dismutase implicated in the defense of floral reproductive tissues. J. Biol. Chem. 2000, 275, 36726-36733.

6. Zimmerman, G.; Bäumlein, H.; Mock, H.P.; Himmelbach, A.; Schweizer, P. The multigene family encoding germin-like proteins of barley. Regulation and function in basal host resistance. Plant Physiol. 2006, 142, 181-192.

7. Dunwell, J.; Gibbings, J.G.; Mahmood, T.; Saqlan Naqvi, S.M. Germin and Germin-like Proteins: Evolution, Structure, and Function. Crit. Rev. Plant Sci. 2008, 27, 342-375.

8. Davidson, R.; Reeves, P.A.; Manosalva, P.; Leach, J.E. Germins: A diverse protein family important for crop improvement. Plant Sci. 2009, 177, 499-510.

9. Nakata, M.; Watanabe, Y.; Sakurai, Y.; Hashimoto, Y.; Matsuzaki, M.; Takahashi, Y.; Satoh, T. Germin-like protein gene family of a moss, Physcomitrella patents, phylogenetically falls into two characteristic new clades. Plant Mol. Biol. 2004, 56, 381-395.

10. Rodríguez-López, M.; Baroja-Fernandez, E.; Zandueta-Criado, A.; Moreno-Bruna, B.; Munoz, F.J.; Akazawa, T.; Pozueta-Romero, J. Two isoforms of a nucleotide-sugar pyrophosphatase/phosphodiesterase from barley leaves (Hordeum vulgare L.) are distinct oligomers of HvGLPl, a germin-like protein. FEBS Lett. 2001, 490, 44-48.

11. Barrera-Pacheco, A.; Joaquín-Ramos, A.; Torres-Pacheco, I.; Gonzalez-Chavira, M.; Perez-Perez, C.; Guevara-Olvera, L.; Guevara-Gonzalez, R.G. Analysis of transcriptional expression induced in Capsicum chinense BG-3821 under conditions of biotic and abiotic stress. Agrociencia 2008, 42, 95-106. 
12. Anaya-Lopez, J.L.; Perez-Mora, E.; Torres-Pacheco, I.; Muñoz-Sanchez, C.I.; Guevara-Olvera, L.; Gonzalez-Chavira, M.; Ochoa-Alejo, N.; Rivera-Bustamante, R.F.; Guevara-Gonzalez, R.G. Inducible gene expression by pepper huasteco virus in Capsicum chinense plants with resistance to geminivirus infections. Can. J. Plant Pathol. 2005, 27, 276-282.

13. van Loon, L.C.; Rep, M.; Peterse, C.M.J. Significance of inducible defense-related proteins in infected plants. Annu. Rev. Phytopathol. 2006, 44, 135-162.

14. Lu, M.; Han, Y.-P.; Gao, J.-G.; Wang, X.-J.; Li, W.-B. Identification and análisis of the germin-like gene family in soybean. BMC Genomics 2010, 11, 620-635.

15. Pan, H.Y.; Whittaker, M.M.; Bouveret, R.; Berna, A.; Bernier, F.; Whittaker, J.W. Characterization of wheat germin (oxalate oxidase) expressed by Pichia pastoris. Biochem. Biophys. Res. Commun. 2007, 356, 925-929.

16. Cassland, P.; Larsson, S.; Nilverbrant, N.O.; Jönsson, L.J. Heterologous expression of barley and wheat oxalate oxidase in an E. coli txB gor double mutant. J. Biotechnol. 2004, 109, 53-62.

17. Woo, E.-J.; Dunwell, J.M.; Goodenough, P.W.; Marvier, A.C.; Pickersgill, R.W. Germin is a manganese containing homohexamer with oxalate oxidase and superoxide dismutase activities. Nat. Struct. Biol. 2000, 7, 1036-1040.

18. Manosalva, P.M.; Davidson, R.M.; Liu, B.; Zhu, X.; Hulbert, S.H.; Leung, H.; Leach, J.E. A germin-like protein gene family functions as a complex quantitative trait locus conferring broad-spectrum disease resistance in rice. Plant Physiol. 2009, 149, 286-296.

19. Cheng, H.-Y.; Song, S.-Q. Species and organ diversity in the effects of hydrogen peroxide on superoxide dismutase activity in vitro. J. Integr. Plant Biol. 2006, 48, 672-678.

20. Federico, M.; Iniguez-Luy, F.; Skadsen, R.; Kaeppler, H. Spatial and temporal divergence of expression in duplicated barley germin-like protein-encoding genes. Genetics 2006, 174, 179-190.

21. Lou, Y.; Baldwin, I.T. Silencing of a germin-like gene in Nicotiana attenuata improves performance of native herbivores. Plant Physiol. 2006, 140, 1126-1136.

22. Godfrey, D.; Able, A.; Dry, I. Induction of a grapevine germin-like protein ( VvGLP3) gene is closely linked to the site of Erysiphe necator infection: A possible role in defense? Mol. Plant-Microbe Interact. 2007, 20, 1112-1125.

23. Kunkel, B.N.; Brooks, D.M. Cross talk between signaling pathways in pathogen defense. Curr. Opin. Plant Biol. 2002, 5, 325-331.

24. Mewis, I.; Appel, H.M.; Hom, A.; Raina, R.; Schultz, J.C. Major signaling pathways modulate Arabidopsis glucosinolate accumulation and response to both phloem-feeding and chewing insects. Plant Physiol. 2005, 138, 1149-1162.

25. Burns, S.M.; Hull, S.I. Comparison of serum resistance by defined lipopolysaccharide mutants and an acapsular mutant of uropathogenic Escherichia coli O75:K5. Infect. Immun. 1998, $66,4244-4253$.

26. Sambrook, J.; Fritsch, E.F.; Maniatis, T. Molecular Cloning: A Laboratory Manual; Cold Spring Harbor Laboratory Press: Cold Spring Harbor, NY, USA, 1989; pp. 122-135.

27. Pantoja-Hernandez, M.A.; Muñoz-Sanchez, C.I.; Guevara-Gonzalez, R.G.; Botello-Alvarez, E.; Gonzalez-Chavira, M.; Torres-Pacheco, I.; Guevara-Olvera, L. Expression of ornithine decarboxylase of Coccidioides immitis in three Escherichia coli strains carrying the lamdba DE3 lysogen and an E. coli EWH319 strain odc ${ }^{-}$null mutant. Biotechnol. Lett. 2004, 26, 75-78. 
28. Dumas, B.; Freysinet, G.; Pallett, K. Tissue-specific expression of germin-like oxalate oxidase during development and fungal infection of barley seedlings. Plant Physiol. 1995, 107, 1091-1096.

29. Beauchamp, C.; Fridovich, I. Superoxide dismutase: Improved assays and an assay applicable to acrylamide gels. Anal. Biochem. 1971, 44, 276-287.

30. Godínez-Hernández, Y.; Anaya-López, J.L.; Díaz-Plaza, R.; González-Chavira, M.M.; Torres-Pacheco, I.; Rivera-Bustamante, R.F.; Guevara-González, R.G. Characterization of resistance to pepper huasteco geminivirus in chili Peppers from Yucatán, México. Hortscience 2001, $36,139-142$.

31. Proteomics/post-translational_modification. Available online: http://expasy.org/tools/proteomics/ post-translational_modification. (Accessed on 15 January 2011).

(C) 2011 by the authors; licensee MDPI, Basel, Switzerland. This article is an open access article distributed under the terms and conditions of the Creative Commons Attribution license (http://creativecommons.org/licenses/by/3.0/). 